\title{
Massless Charged Particles, Naked Singularity, and Generalized Uncertainty Principle in Reissner-Nordström-de Sitter-like spacetime
}

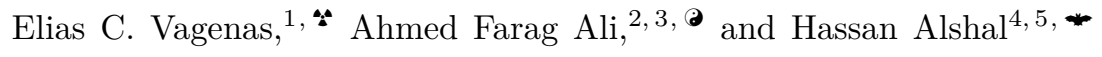 \\ ${ }^{1}$ Theoretical Physics Group, Department of Physics, \\ Kuwait University, P.O. Box 5969, Safat 13060, Kuwait. \\ ${ }^{2}$ Department of Physics, Faculty of Science, Benha University, Benha, 13518, Egypt. \\ ${ }^{3}$ Quantum Gravity Research, Los Angeles, CA 90290, USA \\ ${ }^{4}$ Department of Physics, Faculty of Science, Cairo University, Giza, 12613, Egypt \\ ${ }^{5}$ Department of Physics, University of Miami, Coral Gables, FL 33146, USA
}

\section{Dedication}

One of us (A.F.A.) wishes to dedicate this work to the memory of his late father Farag Mohammad Ali whose sudden death during the preparation of the final draft of this work in 2019 greatly sorrowed him.

\begin{abstract}
Motivated by the endeavors of Li Xiang and You-Gen Shen on naked singularities, we investigate the validity of the cosmic censorship conjecture in the context of generalized uncertainty principle. In particular, upon considering both linear and quadratic terms of momentum in the uncertainty principle, we first compute the entropy of a massless charged black hole in de Sitter spacetime at a given modified temperature. Then, we compute the corresponding modified cosmological radius and express the black hole electric charge in terms of this modified cosmological radius and, thus, in terms of the generalized uncertainty principle parameter. Finally, we examine whether such a system will end up being a naked singularity or it might be protected by the cosmic censorship conjecture, and how that might be related to the possible existence of massless charged particles.
\end{abstract}

\section{INTRODUCTION}

General Relativity (GR) is so successful not only because of its accurate predictions but also its capability of predicting its shortcomings. One of the "apparent" shortcomings of GR is that it predicts the existence of singularities, spacetime geometrical points where curvature becomes infinite and laws of physics are no longer working. Einstein abhorred their occurrence 1]. He believed they are just made-up ramifications of spacetime symmetries and morphisms. However it turned out that they are inescapable through PenroseHawking singularity theorems [2 -5]. It is worth noting that the classical Einstein-Maxwell equations were introduced as a deterministic unified theory. However, it was shown that the Einstein-Maxwell equations can avoid singularities through, interestingly, violating energy density condition of positivity of Penrose - Hawking singularity theorems [6, 7]. Nevertheless, this violation has undesired consequences [8, 9] upon considering Positive Energy Theorem [10 12]. So the moral is that GR, alone or combined with other classical theories, fails to deal with singularities. However, this is not truly considered a shortfall as long as the singularity is causally separated - and hence impossible to be observed - from the rest of the universe through an event horizon, a "doorstep" at which timelike and spacelike coordinates interchange their

¿Electronic address: elias.vagenas@ku.edu.kw

Electronic address: ahmed@quantumgravityresearch.org

- Electronic address: haishai@sci.cu.edu.eg rules. That makes static uncharged black hole singularities spacelike points, meanwhile those of rotating and/or charged black holes may disrupt the causal structure of spacetime. Interestingly since PenroseHawking singularity theorems do not say much about geometrical locations of singularities, then existence of singularity is not necessarily accompanied within black hole structure [13, i.e., they might not be in need of event horizon. These singularities are called naked singularities. Since a naked singularity lacks that "doorstep", timelike and spacelike coordinates keep their geometrical rules unchanged around that point. This would lead to a major breakdown of foundations of spacetime geometry. Consequently, the "realistic" perspectives on classical theories of physics would be demolished. Being a Platonist ${ }^{1}$ [14], Penrose introduced the cosmic censorship conjecture [3]:

"Nature abhors a naked singularity".

Since the introduction of cosmic censorship conjecture, many endeavors attempted to argue in favor of - mainly by focusing on Cauchy horizon [15, 23] - or against the weak and strong versions of cosmic censorship conjecture - mainly by focusing on gravitational dust collapse processes [24 33. None came with conclusive definitive proof whether naked singularities could or could not physically exist, with a quest

\footnotetext{
${ }^{1}$ Hawking meant "Platonic realist". Like Einstein, Penrose concerns about maintaining the deterministic predictability of GR as a local theory. This philosophical stance is based on the fact that GR is exclusively a geometric theory of Lorentzian manifolds.
} 
whether it should be in need for changing the methodology of dealing with cosmic censorship conjecture 34. Another line of research that deals with this conjecture is to see its topological effects together with null energy condition [35, 48]. Also there is another debate about the validity of either cosmic censorship conjecture or naked singularities in modified gravity theories upon considering null energy condition [49 [57]. Recent analytical proof [58] is in favor of cosmic censorship conjecture, while others [59 61] counter-argued cosmic censorship conjecture, leaving the quest unanswered.

The biggest challenge in contemporary physics is to unify GR and Quantum Mechanics (QM) in a concrete theory of quantum gravity. A little bit auspicious way, of many, to seize the "holy grail" is to construct a quantum field theory in curved spacetime [62, 63], where the curved spacetimes are black holes. This approach succeeded in introducing Hawking radiation and black hole entropy [64, 65] and black hole information riddle 66. Before that, Wald's classical gedanken experiment failed to destroy event horizon by overcharging the corresponding Kerr-Newman black hole [67]. If the experiment had succeeded, then-upon considering the quantum effects - the resulting naked singularity would have absorbed all black hole entropy. This would contradict the holographic principle 68, 69] as a naked singularity of Planck length size $\ell_{p}$ can carry only few bits of information. More recent classical gedanken experiments also support cosmic censorship conjecture upon considering different Reissner-Nordström black hole [70] or upon considering the same Kerr-Newman black hole with overcharge and overspin together [71. So within the conservation of information paradigm, "stripping" singularity would provoke vehement information loss [72. It is worth noting that despite the metric at singularity can be no longer regular, i.e., it is degenerate, information may be retrieved out of the singularity even if the used technique does not work out the initial value formulation 73 .

So until the "advent" of a mature, consistent, and complete theory of quantum gravity takes place, the question of cosmic censorship conjecture remains open. But, generally, it is believed that GR and QM "marriage" would happen upon some compromises. It could be necessary that QM laws need some tweaks, e.g., modifying Heisenberg Uncertainty Principle (HUP), to be compatible with a fundamental characteristic of String theory, that is energy corresponds with UV/IR increment in its length. That leads us to introduce a Generalized Uncertainty Principle (GUP) [74 83] as another attempt to reconcile GR and QM.

In the remainder of this work, we summarize the endeavors of Li and Shen [84] as well as of Xiang and Shen 85 on examining the effect of the quadratic GUP on cosmic censorship conjecture. Then, we introduce both linear and quadratic GUP to compute the GUP-modified entropy for a static spherically symmetric black hole in de Sitter spacetime. For the specific case of the massless charged ReissnerNordström-de Sitter (RNdS) spacetime, we find the location of the cosmological horizon and also show that there are no more horizons so the curvature singularity is a naked singularity. Thus, one can assume that the Hawking radiation consists of massless charged particles. Therefore, we calculate the total energy density of those massless charged particles. Finally, the results with some concluding comments are presented. Here, natural units will be used, i.e., $\hbar=c=k_{B}=1$.

\section{QUADRATIC GUP EFFECTS ON RNdS-LIKE SPACETIME}

In this section, we summarize the analysis of Refs. [84, 85] starting by considering the quadratic GUP to be given by

$$
\Delta x \Delta p \geqslant 1+\lambda \Delta p^{2}
$$

which gives an uncertainty in momentum

$$
\Delta p \sim \frac{\Delta x-\sqrt{\Delta x^{2}-4 \lambda}}{2 \lambda}
$$

where $\lambda$ is the dimensionful GUP parameter which is proportional to the squared Planck length $\ell_{p}^{2}$ with $\ell_{p} \sim \sqrt{G}$, and $G$ is Newton's gravitational constant. According to Planckian thermodynamics $p \sim E \sim T$. Therefore, in the presence of a quantum black hole of event horizon radius $r_{h}$, Eq. (2) can be read as

$$
T \sim \frac{r_{h}-\sqrt{r_{h}^{2}-4 \lambda}}{2 \lambda} .
$$

Similarly, for time and energy conjugates we have

$$
\Delta E \sim \frac{\Delta t-\sqrt{\Delta t^{2}-4 \lambda}}{2 \lambda}
$$

where the signs in front of the radicals in Eqs. (2), (3), and (4) were selected such that when we take the $\lambda \rightarrow 0$ limit, we then obtain the conventional HUP

$$
\Delta p \sim \frac{1}{\Delta x} \quad \text { and } \quad \Delta E \sim \frac{1}{\Delta t} .
$$

At this point, it is worth making the following comment. The GUP corrections can be assigned to the Planck constant ${ }^{2}$, i.e., $\hbar$, and thus we can define an effective Planck constant as

$$
\hbar^{\prime} \sim \hbar\left(1+\lambda \Delta E^{2}\right) .
$$

In this case, the Hawking temperature, i.e., $T_{H}=\frac{\hbar}{\beta}$, is modified and becomes

$$
T_{H}^{\prime} \sim \frac{\hbar\left(1+\lambda \Delta E^{2}\right)}{\beta}
$$

\footnotetext{
${ }^{2}$ Here, only for this comment, we have reinstated the units of $\hbar$.
} 
where $\beta$ is called the reciprocal temperature and is given as $\beta=2 \pi \kappa^{-1}$ with $\kappa$ to be the surface gravity of the black hole horizon.

Since the surface gravity is inversely proportional to the black hole radius, i.e., $\kappa \sim r_{h}^{-1}$, the reciprocal temperature will be proportional to the black hole radius, i.e., $\beta \sim r_{h}$, and thus, the temperature given in Eq. (3) becomes

$$
\begin{aligned}
T_{H}^{\prime} & =\frac{\beta-\sqrt{\beta^{2}-4 \lambda}}{2 \lambda} \\
& =\frac{2}{\beta+\sqrt{\beta^{2}-4 \lambda}} .
\end{aligned}
$$

The first law of black hole mechanics [86] for a Schwarzschild black hole in de Sitter space (SdS) 84] reads

$$
d M=-\frac{\kappa_{c}}{8 \pi} d A_{c}-\frac{V}{8 \pi} d \Lambda
$$

where the subscript "c" denotes the cosmological horizon, $\Lambda$ is the cosmological constant, and $V=\frac{4 \pi}{3} r_{c}^{3}$ is the volume of the dS universe. Now due to the problem of negative temperatures (see the factor of the differential $d A_{c}$ in Eq. (10), we redefine $M$ to be the mass of everything inside the cosmological horizon, i.e., $r_{c}$, including the black hole mass. This defines the total energy of such a system to be $E_{0}=M+E_{v a c}$ and we demand this total energy to be conserved. Therefore, the first law of thermodynamics for the cosmological horizon becomes

$$
d E_{v a c}=-d M=\frac{\kappa_{c}}{8 \pi} d A_{c}+\frac{V}{8 \pi} d \Lambda .
$$

When we also demand the entropy of this system to be maximum, then the modified temperature of the black hole, i.e., $T_{H}^{\prime}$, becomes equal to the temperature of the cosmological horizon, i.e., $T_{c}$, and the entropy of cosmological horizon becomes

$$
\begin{aligned}
S_{c} & =\int \frac{d E_{v a c}}{T_{c}} \\
& =\frac{A_{c}}{4}-\int \frac{\lambda}{4 \beta^{2}} d A_{c}
\end{aligned}
$$

where $A_{c}$ is cosmological area. At this point we should point out that if we take the limit $\lambda \rightarrow 0$, we obtain the Bekenstein-Hawking entropy of the cosmological horizon in the context of HUP.

Let us now assume we have a massless charged Reissner-Nordström-de Sitter spacetime (RNdS-like) with metric

$$
d s^{2}=-f(r, Q, \Lambda) d t^{2}+\frac{d r^{2}}{f(r, Q, \Lambda)}+r^{2} d \Omega
$$

with the components of the metric to be

$$
f(r, Q, \Lambda)=1+\frac{Q^{2}}{r^{2}}-\frac{\Lambda r^{2}}{3}
$$

and the cosmological horizon, i.e., $r_{c}$, is defined as $f\left(r_{c}, Q, \Lambda\right)=0$ thus the electric charge reads

$$
Q^{2}=\frac{\Lambda r_{c}^{4}}{3}-r_{c}^{2}
$$

The corresponding surface gravity will be

$$
\kappa_{c}=\left(\frac{2 \Lambda r_{c}}{3}-\frac{1}{r_{c}}\right) \text {. }
$$

Employing Eq. 16 in order to compute the last term of Eq. 12, we get

$$
\begin{aligned}
\Delta S & =-\frac{\lambda}{16 \pi^{2}} \int \kappa^{2} d A_{c} \\
& =-\frac{\lambda}{2 \pi}\left(\ln r_{c}-\frac{2 \Lambda}{3} r_{c}^{2}+\frac{\Lambda^{2}}{9} r_{c}^{4}\right) .
\end{aligned}
$$

Now we can consider the most probable $\Lambda$ associated with the maximum entropy by setting $\partial(\Delta S) / \partial \Lambda=0$. This will give $r_{c}=\sqrt{3 / \Lambda}$ which means that the corresponding cosmological radius is equal to the radius of de Sitter spacetime that is empty of any mass and charge. Therefore, in the context of the quadratic GUP, the second law of black hole mechanics forbids the existence of massless charged particles.

In Ref. 85, it was shown that, in systems such as the one under study here, cosmic censorship conjecture is guaranteed by the quadratic GUP upon considering the energy-time uncertainty

$$
\Delta t \geqslant \frac{1}{\Delta E}+\lambda \Delta E
$$

that imposes a bound on the rate of energy loss as

$$
\frac{\Delta E}{\Delta t} \sim \frac{d E}{d t}<\frac{1}{\lambda}
$$

which in turn leads to

$$
E \leqslant \frac{L}{\lambda} \sim \frac{1}{\sqrt{\lambda}}
$$

where $L$ is characteristic length of the system under study. The reason why Eq. 20 keeps cosmic censorship conjecture safe is that for a "covered" black hole singularity to be a naked singularity, it is necessary the black hole to absorb the total mass of the system which means $E \gg m_{p} \sim \frac{1}{\sqrt{\lambda}}$. It is evident that this contradicts our result given by Eq. 20 in the context of quadratic GUP.

\section{LINEAR AND QUADRATIC GUP EFFECTS ON RNdS-LIKE SPACETIME}

In this section, following the previous analysis we will re-examine everything we have just summarized in [85] in the light of linear and quadratic GUP. The linear and quadratic GUP, which is also compatible with Doubly Special Relativity, is given as [83]

$$
\Delta x \Delta p \geqslant 1-\alpha \Delta p+4 \alpha^{2} \Delta p^{2}
$$

where if the $\alpha$-term is vanished, then if one sets $\lambda=$ $4 \alpha^{2}$, it will get Eq. (1). The corresponding GUPmodified uncertainty in momentum is of the form

$$
\Delta p \sim \frac{(\Delta x+\alpha)-\sqrt{(\Delta x+\alpha)^{2}-16 \alpha^{2}}}{8 \alpha^{2}}
$$


and the GUP-modified uncertainty in energy will now read

$$
\Delta E \sim \frac{(\Delta t+\alpha)-\sqrt{(\Delta t+\alpha)^{2}-16 \alpha^{2}}}{8 \alpha^{2}},
$$

with the corresponding GUP-modified temperature to be of the form

$$
T \sim \frac{\left(r_{h}+\alpha\right)-\sqrt{\left(r_{h}+\alpha\right)^{2}-16 \alpha^{2}}}{8 \alpha^{2}} .
$$

As in the previous section, one can make the comment that all linear and quadratic GUP corrections can be assigned to an effective Planck constant which is now modified as

$$
\hbar^{\prime} \sim \hbar\left(1-\alpha \Delta E+4 \alpha^{2} \Delta E^{2}\right) .
$$

Therefore, the GUP-modified Hawking temperature becomes

$$
\begin{aligned}
T_{H}^{\prime} & =\frac{(\beta+\alpha)-\sqrt{(\beta+\alpha)^{2}-16 \alpha^{2}}}{8 \alpha^{2}} \\
& =\frac{2}{(\beta+\alpha)+\sqrt{(\beta+\alpha)^{2}-16 \alpha^{2}}}
\end{aligned}
$$

which, as expected, is exactly the same with the one given in Eq. (24) since, as already mentioned, $\beta \sim r_{h}$. Now, following the analysis of the previous section, inside the cosmological horizon the modified Hawking temperature, i.e., $T_{H}^{\prime}$, becomes equal to the temperature of the cosmological horizon, i.e., $T_{c}$, the corresponding entropy of the cosmological horizon becomes

$$
\begin{aligned}
S_{c} & =\int \frac{d E_{v a c}}{T_{c}} \\
& =\int \frac{\left.(\beta+\alpha)+\sqrt{(\beta+\alpha)^{2}-16 \alpha^{2}}\right)}{2} \times \frac{\kappa_{c} d A_{c}}{8 \pi} .
\end{aligned}
$$

Then, we expand $S_{c}$ up to $\mathcal{O}\left(\alpha^{3}\right)$ to get

$$
S_{c}=\frac{A_{c}}{4}-\int\left(\frac{\alpha^{2}}{\beta^{2}}-\frac{\alpha}{4 \beta}\right) d A_{c} .
$$

At this point, it should be stressed that this extra $\frac{\alpha}{2 \beta}$ term will dramatically change the previous calculations for the cosmological radius and consequently everything after.

Let us now employ the metric of a massless charged RNdS-like black hole. First, upon combining Eq. 16 and Eq. 28, the GUP-corrected entropy $\Delta S$ ends up being

$$
\begin{aligned}
\Delta S= & -\int\left(\frac{\alpha^{2}}{\beta^{2}}-\frac{\alpha}{4 \beta}\right) d A_{c} \\
= & \int\left[-\frac{\alpha^{2}}{4 \pi^{2}}\left(\frac{4 \Lambda^{2}}{9} r_{c}^{2}-\frac{4 \Lambda}{3}+\frac{1}{r_{c}^{2}}\right)\right. \\
& \left.+\frac{\alpha}{8 \pi}\left(\frac{2 \Lambda}{3} r_{c}-\frac{1}{r_{c}}\right)\right] d A_{c} \\
= & -\frac{2 \alpha^{2}}{\pi}\left(\frac{\Lambda^{2}}{9} r_{c}^{4}-\frac{2 \Lambda}{3} r_{c}^{2}+\ln r_{c}\right) \\
& +\alpha\left(\frac{2 \Lambda}{9} r_{c}^{3}-r_{c}\right)
\end{aligned}
$$

Then, maximizing the entropy with respect to $\Lambda$ in the light of the extra $\alpha$-term in $\Delta S$, the corresponding cosmological radius is determined by both $\alpha$ and $\Lambda$ together according to the equation

$$
r_{c}^{2}-\frac{\pi}{2 \alpha \Lambda} r_{c}-\frac{3}{\Lambda}=0
$$

which gives the root

$$
r_{c}=\left(\frac{\pi}{4 \alpha \Lambda}\right)+\sqrt{\left(\frac{\pi}{4 \alpha \Lambda}\right)^{2}+\frac{3}{\Lambda}} .
$$

It is noteworthy that the other root in order to be positive demands $\Lambda<0$, which of course contradicts the fact that spacetime is de Sitter.

For the sake of comparison with the result obtained in the previous section, namely $r_{c}=\sqrt{3 / \Lambda}$, we expand the root given in Eq. 33 up to $\mathcal{O}\left(\alpha^{3}\right)$ to obtain

$$
r_{c} \sim\left(\frac{\pi}{4 \alpha \Lambda}\right)+\sqrt{\frac{3}{\Lambda}}\left[1+\frac{1}{2}\left(\frac{\pi}{4 \alpha \Lambda}\right)^{2} \frac{\Lambda}{3}\right] .
$$

At this point a couple of comments are in order. First, when we are in strong gravity regimes, e.g. near black hole horizons, which can be viewed as $\alpha \rightarrow \infty$, from Eq. 34 we obtain $r_{c} \rightarrow \sqrt{3 / \Lambda}$ which agrees with what was presented in the previous section and proven in Ref. [85. Second, if we employ Eq. (33), equation $f\left(r_{c}, Q, \Lambda\right)=0$ will be satisfied for an electric charge $Q$ of the form

$$
\begin{aligned}
|Q|=\frac{1}{8 \sqrt{6}} & \left(\frac{4 \pi^{4}}{\alpha^{4} \Lambda^{3}}+\frac{144 \pi^{2}}{\alpha^{2} \Lambda^{2}}\right. \\
& +\frac{\pi\left(48 \alpha^{2} \Lambda+\pi^{2}\right)^{3 / 2}}{\alpha^{4} \Lambda^{3}} \\
& \left.+\frac{3 \pi^{3} \sqrt{48 \alpha^{2} \Lambda+\pi^{2}}}{\alpha^{4} \Lambda^{3}}\right)^{1 / 2} \neq 0 .
\end{aligned}
$$

It is clear that if we take the limit $\alpha \rightarrow \infty$, from Eq. (13) the electric charge will be $Q \rightarrow 0$, as expected.

Furthermore, since the electric charge receives a nonzero value, namely $|Q| \neq 0$, solving equation $f(r, Q, \Lambda)=0$ with $f(r, Q, \Lambda)$ as given by Eq. (14), we get the roots (radii of horizons)

$$
\begin{aligned}
& r_{ \pm \pm}= \pm \sqrt{\frac{3 \pm \sqrt{9+12 Q^{2} \Lambda}}{2 \Lambda}} \\
& r_{ \pm \mp}= \pm \sqrt{\frac{3 \mp \sqrt{9+12 Q^{2} \Lambda}}{2 \Lambda}}
\end{aligned}
$$

Since the radii of the horizons have to be positive, i.e., $r<0$, the negative roots are excluded completely. The root $r_{++}$is the largest one so it is the cosmological horizon [87]. The root $r_{+-}$has also to be positive and, thus, it is required $3-\sqrt{9+12 Q^{2} \Lambda}>0$. However, this gives $Q^{2}<0$ which is impossible thus the root $r_{+-}$is unphysical and is also removed. Therefore, there is no event horizon, and the singularity of the RNdS-like black hole is a naked singularity. 


\section{EFFECT OF LINEAR AND QUADRATIC GUP ON ENERGY DISTRIBUTION OF MASSLESS CHARGED PARTICLES IN RNdS-LIKE SPACETIME}

Assuming that the curvature singularity can store only a few bits of information, one may say that the Hawking radiation for the black hole under study will consist of massless charged particles. Thus, it is useful to compute the total energy density of these massless charged particles.

Following the analysis in Ref. [88, we calculate the total energy density of the massless charged particles in a general spherically symmetric static spacetime in the context of linear and quadratic GUP. The first quantity to be employed for this calculation, is the invariant volume element of the phase space. In Ref. [89, the invariant volume element of a phase space in a $D$-dimensional spacetime was computed in the context of linear GUP, while in Ref. [90, the invariant volume element of a phase space in $D$-dimensional spacetime was computed in the context of linear and quadratic GUP. However, in the latter case, the invariant volume was computed to $\mathcal{O}(\alpha)$ while here we would like to be more precise and, thus, go up to $\mathcal{O}\left(\alpha^{2}\right)$. Therefore, the invariant volume element of a phase space in the context of linear and quadratic GUP to $\mathcal{O}\left(\alpha^{2}\right)$ is given as 91 .

$$
\frac{d \mathbf{x}^{D} d \mathbf{p}^{D}}{(2 \pi)^{D}\left(1-\alpha p+\left(\frac{2 \alpha^{2}}{D+1}+\frac{\alpha^{2}}{2}\right) p^{2}\right)^{(D+1)}} .
$$

At the WKB level, the norm of massless particle momentum 3-space vector is

$$
p^{2}=p_{i} p^{i}=\frac{w^{2}}{f}
$$

where $f=f(r, Q, \Lambda)$ which is given by Eq. (14). Setting $D=3$, the total energy density for all frequencies reads

$$
\rho(f, \beta)=\gamma \int_{0}^{\infty} \frac{f^{2} w^{3}}{2 \pi^{2}\left(f-\alpha \sqrt{f} w+\alpha^{2} w^{2}\right)^{4}} \frac{d w}{\left(e^{\beta w} \pm 1\right)}
$$

where $\gamma$ is the spin degeneracy, the minus stands for the massless charged bosons and the plus stands for massless charged fermions. Upon considering the change of variable $x=\beta w / 2 \pi$ and $T(r)=1 /(\beta \sqrt{f})$, where $T(r)$ is the local temperature, Eq. 40 becomes

$$
\rho(x, T)=8 \pi^{2} \gamma T^{4} \int_{0}^{\infty} \frac{x^{3}}{\left(1-a x+a^{2} x^{2}\right)^{4}} \frac{d x}{\left(e^{2 \pi x} \pm 1\right)}
$$

where $a=2 \pi \alpha T$. This integral is not quite easy to solve. However, at least for the bosons and up to $\mathcal{O}\left(\alpha^{3}\right)$, it looks close to Hurwitz zeta function

$$
\zeta(n, u)=\frac{1}{\Gamma(n)} \int_{0}^{\infty} \frac{x^{n-1} e^{-u x}}{1-e^{x}} d x
$$

where $a, n>0$. In this case, the total density given by Eq. 41) is indeed a convergent integral. Thus, using contour integral techniques, Eq. 41 can be calculated, However, we focus more on demonstrating the effect of GUP on such distribution(s) and, thus, we provide Figs. (1) and (2). For fixed $\alpha$ and $T(r)$, we assume $a$ to be small compared with $x$.

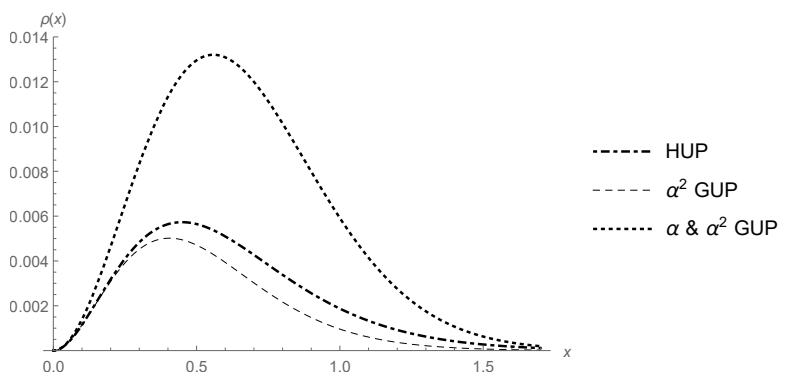

Figure 1: The total energy density $\rho(x)$ versus the variable $x$ for bosons with $a=\frac{1}{2}$.

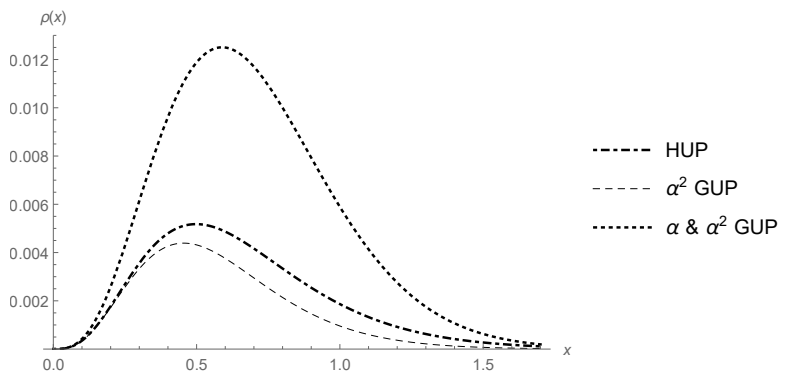

Figure 2: The total energy density $\rho(x)$ versus the variable $x$ for fermions with $a=\frac{1}{2}$.

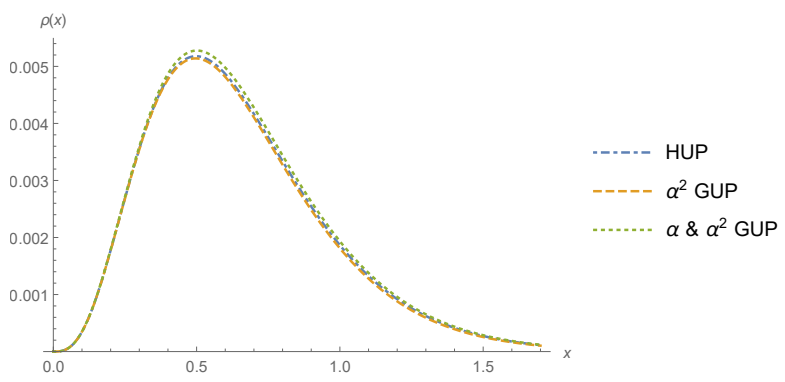

Figure 3: The total energy density $\rho(x)$ versus the variable $x$ for bosons with $a=0.01$

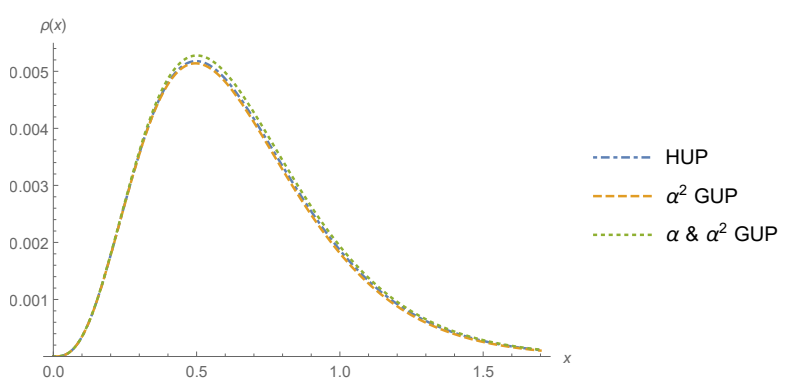

Figure 4: The total energy density $\rho(x)$ versus the variable $x$ for fermions with $a=0.01$.

A number of comments are in order here. First, for very diminutive values of $a$, as in Figs. (3) and (4), 
we notice that the curves of GUP tend to be that of HUP, as expected. Second, when such a collapsing system reaches the state of ultracold black hole, where $T(r) \sim \kappa_{c} \rightarrow 092$, and since the constant $a$ is small only if $\alpha$ is also small, it is evident that we have the GUP to tend to the HUP, as expected. Third, if we keep the GUP parameter $\alpha$ fixed and consider a different metric component $f \sim 1 / T^{2}(r)$, say for instance the $f(M, r, Q, \Lambda)$ of the standard massive RNdS spacetime, to be compared with the larger $f(r, Q, \Lambda)$ of the massless RNdS spacetime for any allowed physical radius, then we notice that the massless one is colder, and hence $a$ is smaller for the massless RNdS spacetime, as expected.

\section{CONCLUSIONS}

In this work we have followed the methodology presented in Refs. 84, 85 except we have introduced a linear term in momentum in the GUP, namely linear and quadratic GUP, in order to investigate the GUP effect on a black hole system. We first computed the GUP-modified temperature and, using the first law of black hole mechanics, the GUP-modified entropy of the cosmological horizon. In our case, the entropy does not only have a quadratic GUP correction term but also a linear GUP correction term. Then, for the specific massless RNdS spacetime, since the modified entropy of the cosmological horizon depends explicitly on the cosmological radius, we calculated the GUPmodified cosmological radius. Moreover, we expressed the electric charge of the specific black hole in terms of the GUP-modified cosmological horizon radius. Since the electric charge is nonzero, the equation for the locations of the black hole horizons is solved. The cosmological horizon is the only physical horizon, and thus it exists while there is no event horizon. Therefore, the singularity, i.e., $r=0$, is a naked singularity and, thus, the cosmic censorship conjecture is violated in the black hole spacetime under study. Furthermore, in Refs. [84, 85], it was also shown that by considering the quadratic GUP, the second law of black hole mechanics prevents the occurrence of massless charged particles. Assuming that the singularity can store only a few bits of information, one may say that the Hawking radiation for the black hole under study will consist of massless charged particles. For this reason, we also compute the total energy density of these massless charged particles in RNdS-like spacetime and in the presence of linear and quadratic GUP. Our result does not say that massless charged particles can exist within the contemporary known Standard Model. Rather, contrary to [85], it says that until we get a concrete theory of quantum gravity, there is no physical principle that prohibits the existence of massless charged particles upon combining the second law of black hole mechanics together with the more general, linear and quadratic GUP. It is also worth noting that if massless charged particles existed in low/moderate energies, they would have been detected easily.

In contrast, this is not contradictory with the most famous hypothesis for massless charged particles where massless quarks are believed to exist at very high energies before symmetry breaking occurred (see for instance Ref. 93]). Despite we discuss an ultracold black hole-like system, we showed that in the presence of linear and quadratic GUP, the corresponding massless charged particles have huge energy density compared with those of HUP and of the quadratic GUP. Even if those tentatively assumed massless charged particles are indeed not comparable to massless quarks in their features or the way of formation, the gigantic effect of gravitational collapse near the fundamental length, that is necessary to form massless charged particles, might be comparable to the high energy condition to form massless quarks.

Finally, we would like to emphasize on the subtlety of both fundamental topics, namely the cosmic censorship conjecture and the massless charged particles, discussed here. It is our belief that the fundamental topics can not be resolved using phenomenological, semiclassical, and/or heuristic methodologies of quantum gravity 94. Thus, we agree with Xiang and Shen on the indispensability of a full theory of quantum gravity theory to be applied when star collapses in order to get the required full picture of such phenomena.
[1] J. Earman, "Bangs, crunches, whimpers, and shrieks: Singularities and acausalities in relativistic spacetimes", Oxford Univ. Pr. (1995).

[2] R. Penrose, Phys. Rev. Lett. 14, 57 (1965).

[3] R. Penrose, Riv. Nuovo Cim. 1, 252 (1969) [Gen. Rel. Grav. 34, 1141 (2002)].

[4] S. W. Hawking and R. Penrose, Proc. Roy. Soc. Lond. A 314, 529 (1970).

[5] S. W. Hawking and G. F. R. Ellis, "The Large Scale Structure of Space-Time", Cambridge Univ. Pr. (2011).

[6] P. Dolan, Commun. Math. Phys. 9, 161 (1968).

[7] F. Finster, J. Smoller and S. T. Yau, Commun. Math. Phys. 205, 249 (1999) gr-qc/9810048.

[8] G. W. Gibbons, S. W. Hawking, G. T. Horowitz and M. J. Perry, Commun. Math. Phys. 88, 295 (1983).

[9] M. Herzlich, J.Geom.Phys. 26. 97 (1998).
[10] R. Schon and S. T. Yau, Commun. Math. Phys. 65, 45 (1979).

[11] R. Schon and S. T. Yau, Commun. Math. Phys. 79, 231 (1981).

[12] E. Witten, Commun. Math. Phys. 80, 381 (1981).

[13] G. Srinivasan, "From White Dwarfs to Black Holes: The Legacy of S. Chandrasekhar", The Univ. of Chicago Pr. (1999).

[14] S. Hawking and R. Penrose, "The Nature of space and time", Princeton, USA: Univ. Pr. (1996) 141 p.

[15] M. Simpson and R. Penrose, Int. J. Theor. Phys. 7, 183 (1973).

[16] J. M. McNamara, Proceedings of the Royal Society A bf 358, 499 (1978).

[17] S. Chandrasekhar and J. B. Hartle, Proceedings of the Royal Society A bf 384, 301 (1982).

[18] A. Krolak, Class. Quant. Grav. 3, 267 (1987). 
[19] F. Mellor and I. Moss, Phys. Rev. D 41, 403 (1990).

[20] F. Mellor and I. Moss, Class. Quant. Grav. 9, L43 (1992).

[21] P. R. Brady and E. Poisson, Class. Quant. Grav. 9, 121 (1992)

[22] R. G. Cai and R. K. Su, Phys. Rev. D 52, 666 (1995).

[23] R. M. Wald, In *Iyer, B.R. (ed.) et al.: Black holes, gravitational radiation and the universe* $69-85$ grqc/9710068

[24] P. Yodzis, H. J. Seifert and H. Müller zum Hagen, Commun. Math. Phys. 34, no. 2, 135 (1973)

[25] P. Szekeres, Lect. Notes Phys. 124, 477 (1979).

[26] A. Ori and T. Piran, Phys. Rev. D 42, 1068 (1990).

27] P. Chrusciel, Annals Phys. 202, 100 (1990).

[28] S. L. Shapiro and S. A. Teukolsky, Phys. Rev. Lett. 66, 994 (1991).

[29] M. W. Choptuik, Phys. Rev. Lett. 70, 9 (1993).

[30] D. Christodoulou, Annals Math. 140, 607 (1994).

[31] P. S. Joshi and T. P. Singh, Gen. Rel. Grav. 27, 921 (1995).

[32] D. Garfinkle, Phys. Rev. D 56, 3169 (1997) grqc/9612015.

[33] T. Harada, Pramana 63, 741 (2004) gr-qc/0407109.

[34] R. Penrose, J. Astrophys. Astron. 20, 233 (1999).

[35] P. S. Joshi and R. V. Saraykar, Phys. Lett. A 120, 111 (1987)

[36] M. S. Morris, K. S. Thorne and U. Yurtsever, Phys. Rev. Lett. 61, 1446 (1988).

[37] B. Grubisic and V. Moncrief, Phys. Rev. D 47, 2371 (1993) gr-qc/9209006.

[38] J. L. Friedman, K. Schleich and D. M. Witt, Phys. Rev. Lett. 71, 1486 (1993) Erratum: [Phys. Rev. Lett. 75, 1872 (1995)] gr-qc/9305017].

[39] K. Schleich and D. M. Witt, gr-qc/9903061

[40] T. Jacobson and S. Venkataramani, Class. Quant. Grav. 12, 1055 (1995) gr-qc/9410023.

[41] G. A. Burnett, Phys. Rev. D 52, 6856 (1995) grqc/9504012.

[42] G. Galloway and E. Woolgar, Class. Quant. Grav. 14, L1 (1997) gr-qc/9609007.

[43] G. J. Galloway, K. Schleich, D. M. Witt and E. Woolgar, Phys. Rev. D 60, 104039 (1999) gr-qc/9902061.

[44] G. J. Galloway, K. Schleich, D. Witt and E. Woolgar, Phys. Lett. B 505, 255 (2001) hep-th/9912119.

[45] C. Helfgott, Y. Oz and Y. Yanay, JHEP 0602, 025 (2006) hep-th/0509013.

[46] S. Krasnikov, Grav. Cosmol. 19, 54 (2013) arXiv:1007.4167] [gr-qc]].

[47] H. Ringstrom, Living Rev. Rel. 13, 2 (2010).

[48] E. Curiel, Einstein Stud. 13, 43 (2017) arXiv:1405.0403 [physics.hist-ph]].

[49] K. S. Bhamra, A. M. Finkelstein, V. I. Kreinovich and L. E. Gurevich, Astrophys. Space Sci. 57, 371 (1978).

[50] C. Martinez, J. P. Staforelli and R. Troncoso, Phys. Rev. D 74, 044028 (2006) hep-th/0512022.

[51] K. A. Bronnikov and M. S. Chernakova, Grav. Cosmol. 13, 51 (2007) gr-qc/0703107.

[52] N. Bedjaoui, P. G. LeFloch, J. M. Martin-Garcia and J. Novak, Class. Quant. Grav. 27, 245010 (2010) arXiv:1008.4238 [gr-qc]].

[53] T. L. Curtright, Phys. Lett. B 716, 366 (2012) arXiv:1208.1205 [hep-th]].

[54] C. Charmousis, B. Gouteraux and E. Kiritsis, JHEP 1209, 011 (2012) arXiv:1206.1499 [hep-th]].

[55] P. Rudra, R. Biswas, U. Debnath, R. Biswas and U. Debnath, Astrophys. Space Sci. 354, no. 2, 2101 (2014) arXiv:1307.6603 [physics.gen-ph]].

[56] V. A. Rubakov, Phys. Usp. 57, 128 (2014) [Usp. Fiz. Nauk 184, no. 2, 137 (2014)] arXiv:1401.4024 [hep- th]].

[57] D. A. Tretyakova, B. N. Latosh and S. O. Alexeyev, Class. Quant. Grav. 32, no. 18, 185002 (2015) arXiv:1504.06723 [gr-qc]].

[58] J. C. Del Águila and T. Matos, Class. Quant. Grav. 36, no. 1, 015018 (2019) arXiv:1806.03747 [gr-qc]].

[59] P. Figueras, M. Kunesch and S. Tunyasuvunakool, Phys. Rev. Lett. 116, no. 7, 071102 (2016) arXiv:1512.04532 [hep-th]].

[60] T. Crisford and J. E. Santos, Phys. Rev. Lett. 118, no. 18, 181101 (2017) arXiv:1702.05490 [hep-th]].

[61] M. Dafermos and J. Luk, arXiv:1710.01722 [gr-qc].

[62] N. D. Birrell and P. C. W. Davies, "Quantum Fields in Curved Space" (Cambridge Monographs on Mathematical Physics), Cambridge Univ. Pr. (1984).

[63] L. E. Parker and D. Toms, "Quantum Field Theory in Curved Spacetime : Quantized Field and Gravity" (Cambridge Monographs on Mathematical Physics), Cambridge Univ. Pr. (2009).

[64] S. W. Hawking, Nature 248, 30 (1974).

[65] S. W. Hawking, Commun. Math. Phys. 43, 199 (1975) Erratum: [Commun. Math. Phys. 46, 206 (1976)].

[66] S. W. Hawking, Phys. Rev. D 14, 2460 (1976).

[67] R. M. Wald, Annals Phys. 82, 548 (1974).

[68] G. 't Hooft, Conf. Proc. C 930308, 284 (1993) grqc/9310026.

[69] L. Susskind, J. Math. Phys. 36, 6377 (1995) hepth/9409089

[70] V. E. Hubeny, Phys. Rev. D 59, 064013 (1999) grqc/9808043.

[71] J. Sorce and R. M. Wald, Phys. Rev. D 96, no. 10, 104014 (2017) arXiv:1707.05862 [gr-qc]].

[72] S. D. Mathur, Class. Quant. Grav. 26, 224001 (2009) arXiv:0909.1038 [hep-th]].

[73] O. C. Stoica, J. Phys. Conf. Ser. 626, no. 1, 012028 (2015).

[74] G. Veneziano, Europhys. Lett. 2, 199 (1986).

[75] D. J. Gross and P. F. Mende, Nucl. Phys. B 303, 407 (1988).

[76] D. Amati, M. Ciafaloni and G. Veneziano, Phys. Lett. B 216, 41 (1989).

[77] K. Konishi, G. Paffuti and P. Provero, Phys. Lett. B 234, 276 (1990).

[78] M. Maggiore, Phys. Lett. B 304, 65 (1993) hepth/9301067.

[79] L. J. Garay, Int. J. Mod. Phys. A 10, 145 (1995) grqc/9403008

[80] F. Scardigli, Phys. Lett. B 452, 39 (1999) hepth/9904025.

[81] R. J. Adler, P. Chen and D. I. Santiago, Gen. Rel. Grav. 33, 2101 (2001) gr-qc/0106080.

[82] S. Das and E. C. Vagenas, Phys. Rev. Lett. 101, 221301 (2008) arXiv:0810.5333 [hep-th]].

[83] A. F. Ali, S. Das and E. C. Vagenas, Phys. Lett. B 678, 497 (2009) arXiv:0906.5396 [hep-th]].

[84] X. Li and Y. G. Shen, Phys. Lett. A 324, 255 (2004) hep-th/0308165.

[85] L. Xiang and Y. G. Shen, Mod. Phys. Lett. A 20, $1823(2005)$.

[86] J. M. Bardeen, B. Carter and S. W. Hawking, Commun. Math. Phys. 31, 161 (1973).

[87] M. P. Hobson, G. P. Efstathiou and A. N. Lasenby, "General relativity: An introduction for physicists," Cambridge, UK: Univ. Pr. (2006) 572 p.

[88] Z. H. Li, Phys. Rev. D 80, 084013 (2009).

[89] L. N. Chang, D. Minic, N. Okamura and T. Takeuchi, Phys. Rev. D 65, 125028 (2002) hep-th/0201017.

[90] A. F. Ali, Class. Quant. Grav. 28, 065013 (2011) arXiv:1101.4181 [hep-th]]. 
[91] E. C. Vagenas, A. F. Ali, M. Hemeda and H. Alshal, arXiv:1903.08494 [hep-th].

[92] R. G. Cai, J. Y. Ji and K. S. Soh, Class. Quant. Grav. 15, 2783 (1998) gr-qc/9708062.

[93] H. Fritzsch and M. Gell-Mann, "50 years of quarks", World Scientific (2015).

[94] E. C. Vagenas, A. F. Ali and H. Alshal, arXiv:1811.06614 [gr-qc]. 